\title{
A 5.6 GHz UWB Position Measurement System
}

\author{
Alessandro Cazzorla, Guido De Angelis, Senior Member, IEEE, Antonio Moschitta, Member, IEEE, \\ Marco Dionigi, Member, IEEE, Federico Alimenti, Senior Member, IEEE, \\ and Paolo Carbone, Senior Member, IEEE
}

\begin{abstract}
This paper describes the design and realization of a 5.6 $\mathrm{GHz}$ ultra-wide bandwidth based position measurement system. The system has been entirely made using off-theshelf components and achieves centimeter level accuracy in an indoor environment. It is based on asynchronous modulated pulse round-trip-time measurements. Both system level and realization details are described along with experimental results including estimates of measurement uncertainties.
\end{abstract}

\section{INTRODUCTION}

$\mathbf{T}$ HE topic of indoor positioning has gained increasing interest over the years in the scientific literature because of the involved technical challenges and of the demand in the application market. While it can be envisioned that portable electronic devices will feature seamless outdoor/indoor positioning capabilities in the future, current technology does not enable it yet at acceptable cost-performance trade-offs. Several approaches have been proposed over time that addressed the positioning problem from various perspectives but, even though requests for applications are growing at a fast pace, technology still seems to lay behind. The interested reader is pointed to [1], in which an updated state-of-the art analysis on the available techniques and their accuracy-vs-range figures is published.

The main challenges in the radio indoor positioning field are the high accuracy level required by the majority of the applications and the features of the indoor propagation channel [2], [3]. In this context, pulse-based Ultra-WideBand (UWB) systems are interesting, mainly due to the fine time resolution provided by sub-nanosecond pulses [4]-[6]. This feature enables a high level of accuracy in measuring distance, potentially in the centimeter order. Furthermore, it allows for robustness to multipath phenomena which represent a major challenge of the indoor radio propagation channel [7]. Various researches consider UWB-based positioning, under different viewpoints. These include channel modeling, estimation of multipath components and evaluation of the Cramér-Rao Lower Bound on positioning accuracy, under given conditions [8]-[12].

While CMOS VLSI stands as an enabling technology to attack the positioning problem starting from basic building blocks [13], few complete realizations based on Commercial Off-The-Shelf (COTS) components are described in the literature and even fewer do not use external high-end and standalone numerical instruments. This work describes results ob-

All authors are with the Department of Electrical and Information Engineering, University of Perugia, Italy, PG, 06125 e-mail: alessandrocazzorla@alice.it; \{guido.deangelis; moschitta; dionigi; alimenti; carbone@diei.unipg.it.\}

Manuscript sent May 31, 2011 tained with a positioning system entirely realized using COTS components to reduce complexity and implementation costs. It proposes a simple architecture that extends the state-of-theart in this field [14] by proving experimentally that accurate positioning can be achieved by avoiding the need for complex and expensive solutions. In fact, recently published positioning methods rely on the synchronization of anchors or on the usage of hybrid Direction of Arrival-Time of Arrival (DOA-TOA) approaches or on the usage of complex high speed correlators and digital processing, as published in [15]-[18]. At the same time, because of the adopted measurement method and of the chosen frequency band, bulky antenna arrays as those used in [19] may be avoided. It is also worth noting that the proposed technique may be competitive, from an accuracy standpoint, with recently developed commercial solutions such as [20], that feature $15 \mathrm{~cm}$ ranging error standard deviation under optimal conditions.

Experimental outcomes described in this paper are based on a previously developed ranging system described in [21]. The only common subsystem shared by the two implementations is the Digital Control Unit (DCU), while transceivers including antennas, have been re-designed to work at $5.6 \mathrm{GHz}$ center frequency and new digital signal processing has been adopted to improve position estimation capabilities. Localization is achieved by using Round-Trip-Time (RTT) measurements between master and slave nodes, to further reduce complexity by avoiding the synchronization of anchors. Lack of synchronization requirements is traded-off in this case for the determination and compensation of latencies introduced by the responder devices. This implies the need for system calibration that is not seen as an issue because of the possibility to largely automate this process. Experimental results described in section IV show that state-of-the-art accuracy can be achieved while keeping low circuital complexity.

\section{SySTEM DESIGN}

The design of an UWB-based position measurement system implies competencies from many engineering fields, including metrology, radio-frequency, digital signal processing and system engineering, on the basis of a truly trans- and interdisciplinary approach. The position measurement architecture described in this paper is based on a network of transceivers, in which a master device is located by measuring the RTT from slave nodes located at known positions, as shown in Fig. 1. Each node includes a DCU and a radio-frequencytransceiver. While each slave is designed so as to transmit a pulse when it receives a pulse, with a possibly constant and systematic delay, the master node also includes fine-resolution 
RTT measurement capabilities, by featuring a Time-to-Digital Converter (TDC), characterized by a sub-nanosecond time quantization step. The master node initiates the measurement process by addressing, in turn, the slave devices needed to obtain the 3 RTT measurements. RTT data are then converted into distances by means of calibration information pertinent to each slave node and planar localization is finally achieved. The DCU is a microprocessor-based subsystem that performs management of other electronic resources (e.g. TDC) and enables/disables the transceiver to avoid blinding the detector when a pulse is transmitted. Other design details on this subsystems are published in [21].

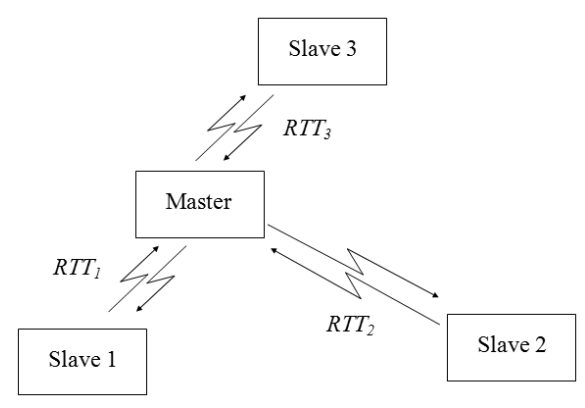

Fig. 1. Measurement principle of the UWB-based localization system.

\section{A. Design of the Transceiver}

The transceiver's role is that of sending and receiving an UWB pulse when triggered by the DCU. The transceivers are identical for both the master and the slave nodes. In order to exploit the regulated UWB frequency band between $4-8 \mathrm{GHz}$, a homodyne architecture has been adopted as illustrated in Fig. 2. The overall system configuration is conceived in order to minimize circuit complexity, component count and fabrication costs. As a consequence a number of advanced radar techniques, such as pulse compression [22] and matched filters [23] have not been considered in this implementation. While these techniques are well-known to perform efficiently under the considered system constraints, their usage at the frequencies considered in this implementation would make it unrealizable using sole COTS components.

To describe the system operation let us consider the master node, the slave node being similar. The distance measurement phase is started by the DCU (not shown in Fig. 2) that initiates (resets) its TDC and sends a triggering signal to the UWB generator. As a consequence a short time pulse is produced in the base-band. The pulser exploits a criticalpath based delay generator made by an ultra-fast AND port and a Schmitt trigger inverter. When triggered, it produces a sub-nanosecond pulse, with $\mathrm{GHz}$ bandwidth. With respect to data published in [21], the pulser used in this realization is based on new AHCMOS digital ports and produces same rise-times but higher voltage swings [24], [25]. The pulse is then used to modulate the oscillator $O_{1}$ [26] in amplitude, that works at $f_{0}=5.6 \mathrm{GHz}$, in free-running mode. The amplitude modulation is obtained by means of a doublebalanced diode mixer $\left(M_{1}\right)$ with high radio-frequency-to- local oscillator isolation [27]. This parameter is important since it is necessary to avoid carrier leakage when the UWB pulse generator is off. After this step the modulated pulse is amplified by the Power Amplifier (PA) [28] and transmitted with a wide-band antenna.
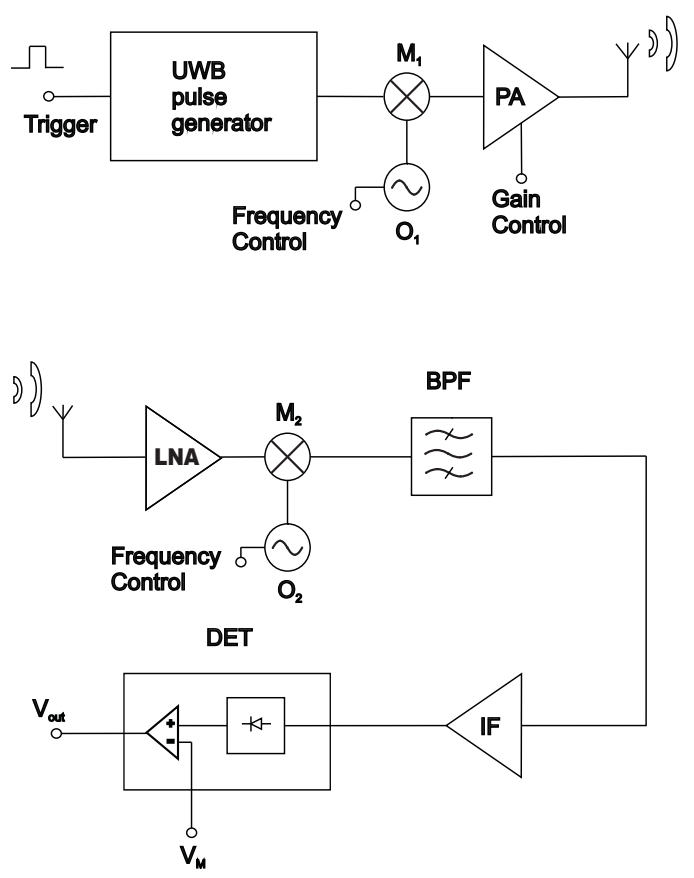

Fig. 2. Block diagram of the analog UWB transceiver front-end. The circuit is based on an homodyne architecture. The two oscillators (O1 and $\mathrm{O} 2)$ are free-running at (approximately) the same $f_{0}=5.6 \mathrm{GHz}$ frequency.

The pulse is received and re-transmitted, after a certain delay, by the slave node. The master node receives the retransmitted pulse. To this purpose a wide-band antenna, identical to that used in the transmission channel, delivers the received power to two low-noise amplifiers in cascade [29], having each a gain of $15 \mathrm{~dB}$, followed by another double-balanced diode mixer $\left(M_{2}\right)$. This mixer is driven by a separate oscillator $\left(\mathrm{O}_{2}\right)$ working in free-running mode at about the same frequency $f_{0}$ as $O_{1}$, the difference in frequency being due to tolerances and environmental factors. The use of two separate oscillators, one for transmission and one for reception, has been required in this prototype to provide as much isolation as possible between the transmission and reception channels.

After the conversion to base-band, the signal is processed by a low-pass filter with enough bandwidth to well accommodate the pulse spectrum. A two-stage Intermediate Frequency (IF) amplifier chain is then used to increase the signal amplitude. Because of the wide signal bandwidth, $\mathrm{SiGe}$ Darlington amplifiers with internal resistive feedback [30] have been adopted to this purpose. Each amplifier stage is matched to $50 \Omega$ and is characterized by a small-signal gain of about $20 \mathrm{~dB}$.

Finally an integrated diode detector [31] is used to reveal the presence of the received pulse. This circuit is composed by a square-law (i.e. energy) detector integrated together with a high-speed comparator. The comparator output is a rectangular pulse, the rising edge of which is used to determine the 
arrival time. The comparator threshold can be set by means of a suitable device pin which, in the present application, is driven by the DCU. Clearly this setting allows trading receiver sensitivity with error probability. The RTT measurement is finally performed by the on-board TDC [32].

From the design point of view a special attention has been paid to power consumption minimization. Another important system design objective was the determination of the right IF gain. This gain has been set to have the receiver noise floor (i.e. the front-end output power without input signal) at exactly the detector threshold power $P_{D}^{T H}$. This power is the minimum input power required by the detector to overcome its internal noise and is equal to about $-20 \mathrm{dBm}$. The overall receiver gain $G_{R X}$ has thus been set to:

$$
G_{R X}=\frac{P_{D}^{T H}}{k T_{0} F_{R} B_{B P F}} \simeq 52 \mathrm{~dB}
$$

where $k$ is the Boltzmann constant, $T_{0}=290 \mathrm{~K}$ is the IEEE standard temperature, $F_{R}$ is the receiver noise figure and $B_{B P F}$ is the equivalent noise bandwidth of the band-pass filter. Using simple assumptions we estimated $F_{R} \approx 3.5 \mathrm{~dB}$ and $B_{B P F} \approx 800 \mathrm{MHz}$. The IF gain $G_{I F}$ can thus immediately be derived as:

$$
G_{I F}=\frac{G_{R X}}{G_{L N A} G_{M_{1}} G_{B P F}} \simeq 35 \mathrm{~dB}
$$

where $G_{L N A}, G_{M_{1}}, G_{B P F}$ are the low-noise-amplifier, mixer M1 and band-pass-filter gains, respectively. The resulting UWB receiver sensitivity amounts to $-61 \mathrm{dBm}$.

The realized prototype is shown in Fig. 3. It is made on a low-cost FR4 printed circuit board along with microstrip interconnection technology. The overall components cost is estimated to be around $\$ 50$ (year 2012) and can further be reduced by properly engineering the circuit. The overall power consumption is about $2.5 \mathrm{~W}$ at $5 \mathrm{~V}$ supply, mainly due to the power amplifiers. Devices made commercially available after the end of this research already require less power, while keeping the same electrical parameters.

The measured transmitted pulse is shown in Fig. 4. Measurement results have been obtained using an equivalenttime numerical sampling oscilloscope with $20 \mathrm{GHz}$ analog bandwidth and $10 \mathrm{Msample/s} \mathrm{sampling} \mathrm{rate.} \mathrm{Consequently,} \mathrm{the}$ signal envelope in Fig. 4 is more significant than fine details. While Fig. 4(A) shows the pulse generated in the baseband, in Fig. 4(B) measurement results are shown after modulation with the $5.6 \mathrm{GHz}$ sinusoidal source, using a fixed $10 \mathrm{~dB}$ attenuator. This latter figure shows that the attenuated peakto-peak value is equal to $530 \mathrm{mV}$ and that a good suppression of the carrier is obtained when the pulse generator is off (i.e. $90 \mathrm{mV}$ residual amplitude). To appreciate the pulse frequency content, spectra are graphed in Fig. 5. Data have obtained using a $20 \mathrm{~dB}$ attenuator and show the baseband and bandshifted behavior of the generated pulse. The carrier is clearly seen around 5.6 GHz. By following the approach taken in [33], the single-shot spatial resolution, $d r$, can be estimated using the expression:

$$
d r=c \frac{t_{R}}{0.7} \simeq 12.8 \mathrm{~cm}
$$
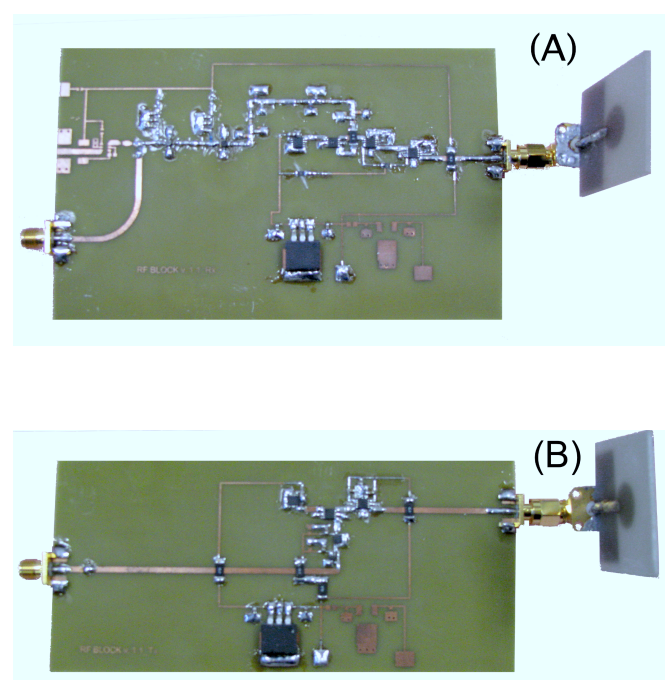

Fig. 3. Fabricated UWB transceiver proptotype operating at $5.6 \mathrm{GHz}$. (A) Receiver, (B) Transmitter.

where $c$ is the speed-of-light in vacuum and $t_{R} \simeq 0.3 \mathrm{~ns}$ is the main pulse rise-time. Thus, as per this design, a singleshot resolution of about $13 \mathrm{~cm}$ is achieved and a much better figure is expected when measurement results are averaged, as shown in sec. IV.
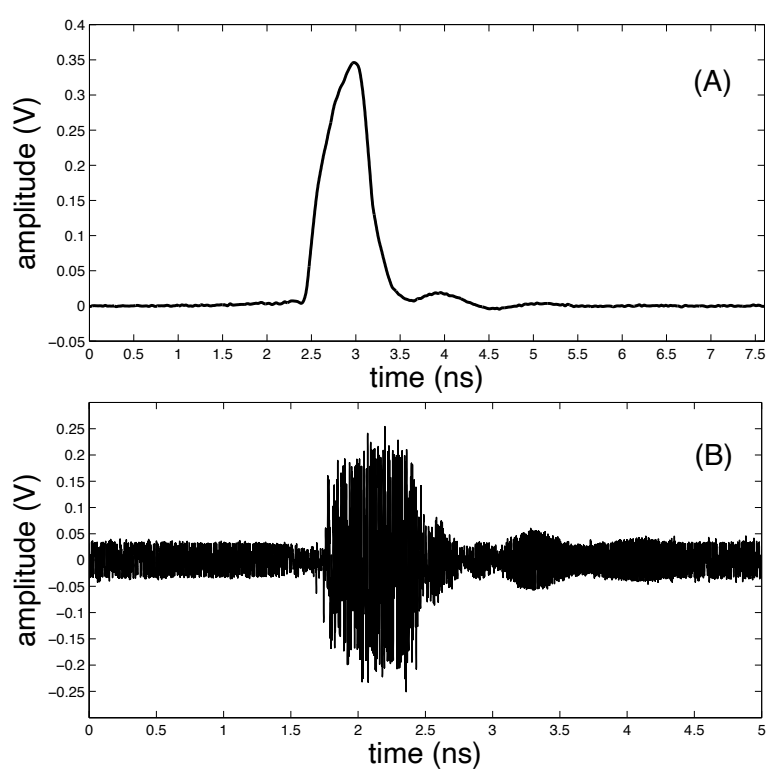

Fig. 4. UWB pulse measurements obtained using a sampling oscilloscope with $20 \mathrm{GHz}$ analog bandwidth, $10 \mathrm{MSample/s}$ sampling rate. (A) Baseband generated UWB pulse (20 dB attenuator inserted).; (B) $5.6 \mathrm{GHz}$ modulated pulse with peak-to-peak value $530 \mathrm{mV}$ and rise-time about equal to $0.3 \mathrm{~ns}$ (10 dB attenuator inserted). 

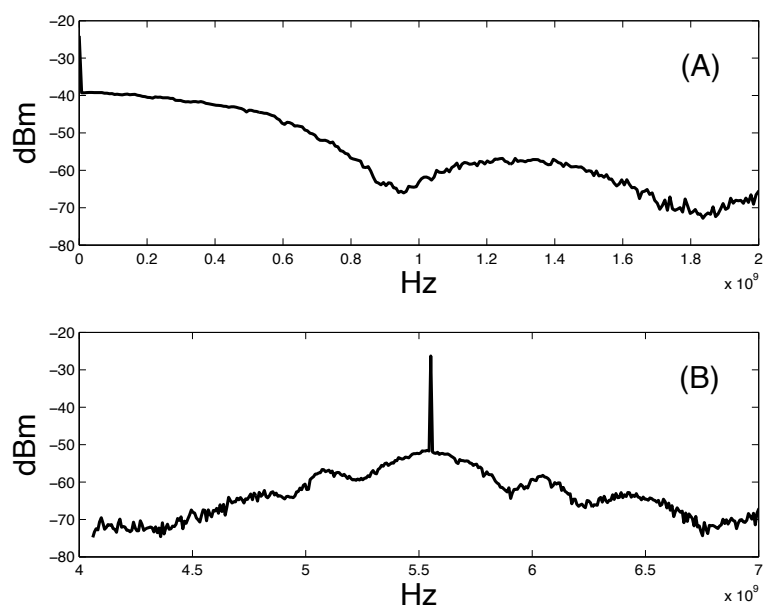

Fig. 5. UWB pulse spectral measurements results. (A) Baseband generated UWB pulse; (B) $5.6 \mathrm{GHz}$ modulated pulse (20 dB attenuator inserted).

\section{B. Design of the Antenna}

The antenna design guideline was the good matching in the signal band and a proper antenna radiation pattern. This latter parameter is of great importance, since anisotropy in emitted power results in modifications of RTT by the radio-frequency detector, beyond what can be recovered using system calibration. We choose an ultra-wideband monopole in order to achieve a compact size and omnidirectional behavior in the azimuthal plane (i.e. around the antenna vertical axes). There are many different UWB monopole antennas in literature, one of the most known being that introduced in [34]. It is composed of a circular printed disk fed directly by a microstrip line. A scheme of the antenna layout is shown in Fig. 6. This design is a modification of the conventional circular disk monopole, obtained by adding a via hole that allows a better matching of the antenna [35]. The antenna has been designed with a disc diameter of $12 \mathrm{~mm}, \mathrm{D}=1.4 \mathrm{~mm}$ and $\mathrm{G}=0.2$ $\mathrm{mm}$ on FR4 substrate $1.6 \mathrm{~mm}$ thick. Fig. 6 shows the antenna schematic and assembly, including the SubMiniatur versionA (SMA) connector, and figure Fig. 7 shows the measured reflection coefficient at the SMA port, that confirms the good matching in the 5-7 GHz band and a bandwidth in excess of 2 GHz. Measurement results shown in Fig. 7 have been taken using a network analyzer with $10 \mathrm{MHz}-40 \mathrm{GHz}$ bandwidth, previously calibrated for measuring the $S_{11}$ parameter [36].

\section{Position Estimation}

Localization on a plane based on RTT requires distance measurements from 3 slaves of known position. When the communication channel is noiseless the RTT characterizing the communication between the master and the $i$-th slave can be approximately modeled as:

$$
t_{i}=\frac{2 \delta_{i}}{v}+\gamma_{i} \quad i=1,2,3
$$

where $\delta_{i}$ is the unknown distance between the master and $i$ th slave, $v$ is the pulse apparent speed, keeping into account both propagation delay and distance dependent contributions

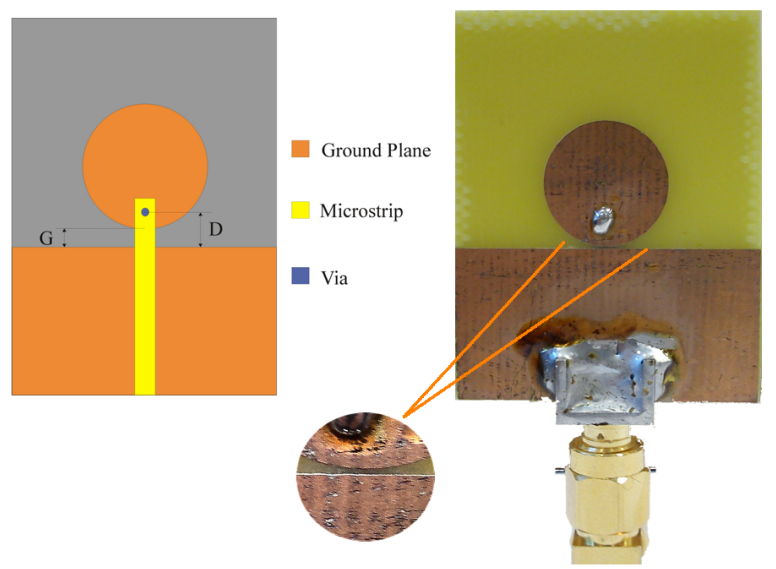

Fig. 6. The UWB antenna layout scheme (left) and its actual implementation (right). The detail of the gap $G$ is enlarged in the inset.

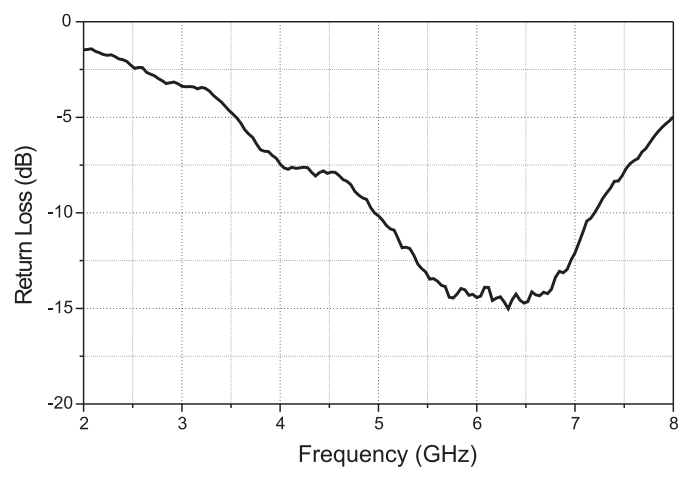

Fig. 7. Measured reflection coefficient $\left(S_{11}\right)$ of the antenna.

to the detection latency, assumed linear [21]. Moreover $\gamma_{i}$ is the delay introduced by the hardware needed to receive and echo this pulse in the $i$-th slave and to transmit and receive the pulse on the master's side. Accordingly, since it depends on device tolerances and environmental factors, the DCU is designed so to minimize the number of components in the signal path. Equation (3) can be inverted to obtain $\delta_{i}$ from a measurement of $t_{i}$, as follows:

$$
\delta_{i}=\frac{v}{2}\left(t_{i}-\gamma_{i}\right) \quad i=1,2,3
$$

that outlines a linear relationship between RTT and distance measurement, in which $v$ and $\gamma_{i}$ are unknowns for a given measurement setup. To estimate these values calibration is performed beforehand, by putting the master at known distances from the $i$-th slave and by measuring the corresponding values of $t_{i}$ [37]. Outcomes in the calibration of the 3 slaves in our system are shown in Fig. 8. Data are obtained by moving the slaves on a linear trajectory with respect to the master put at a fixed distance and by recording the corresponding RTTs. Linear fitting is finally performed. Even though calibration is performed along one direction only, accuracy performance is not degraded as it will be shown in section IV. It can be observed that the RTTs increase approximately linearly in the considered range with slopes and intercepts displayed in Tab. I. These values have been obtained by using the three slave devices and by averaging $N=5 \cdot 10^{3}$ measurement results 
for each calibration point. The nominal slope corresponding to the speed-of-light in vacuum is shown in the rightmost column of Tab. I for reference purposes.

Because of free-space path-loss, wide-band noise, multipath, energy detector dependent effects, asymmetry in the position of transceivers in a node and environmental factors, slopes in Fig. 8 are slightly different and do not match if $v$ is considered to be the speed of light in vacuum. As a result, a delay characterizes the propagation of the pulse through the master-slave setup. This delay is almost linear with distance and is modeled in (3) using the apparent pulse velocity $v$, that becomes numerically larger than the speed of light, as shown by data in Tab. I. Modeling of this phenomenon is published in [21].

Once calibration parameters are known, (4) is used to obtain $\delta_{i}$ from measured values of $t_{i}, i=1,2,3$. These are obtained by addressing each slave in turn, using an 8 -bit pulse position modulation scheme in a preamble of the measurement phase, so that at any time, only a single slave responds to received pulses. Using distances from the 3 slaves, a Non-linear LeastSquare estimator (NLS) provides the planar coordinates of the master. In fact, by defining $\boldsymbol{\theta}=\left[\begin{array}{ll}x & y\end{array}\right]^{T}$ as the column vector of the unknown coordinates, the distance $\delta_{i}$ between the master and the $i-t h$ slave becomes equal to:

$$
\delta_{i}=\sqrt{\left(x-x_{i}\right)^{2}+\left(y-y_{i}\right)^{2}} \quad i=1,2,3
$$

Since single measurements provide limited estimation accuracy, several RTT measurements are taken for each position of the master. Given the characteristics of the used TDC and the moderate dynamic of many practical applications regarding positioning, real-time performance is not compromised. Thus, RTT measurement results are calibrated and numerically averaged in each record and for each slave, before the application of the NLS algorithm. By calling $d_{i}, i=1,2,3$ the resulting mean value and by defining $\boldsymbol{D}=\left[\begin{array}{lll}d_{1} & d_{2} & d_{3}\end{array}\right]^{T}$, NLS can be adopted to find the value $\hat{\boldsymbol{\theta}}$ of $\boldsymbol{\theta}$ that minimizes the summation of squared errors [38]:

$$
J=[\boldsymbol{D}-\boldsymbol{\Delta}(\boldsymbol{\theta})]^{T}[\boldsymbol{D}-\boldsymbol{\Delta}(\boldsymbol{\theta})]
$$

where

$$
\Delta(\boldsymbol{\theta})=\left[\begin{array}{lll}
\delta_{1} & \delta_{2} & \delta_{3}
\end{array}\right]^{T} .
$$

The NLS cycles over a fixed number of iterations. Repeated empirical tests suggested that 60 cycles are enough for the algorithm to converge.

Notice that experimental results given by practical implementations such as this one, highlight a certain number of behaviors that are not modeled by (3): pulse shape modifications and widening, effects due to shadowing and multipath, anisotropic responses by antennas, non-idealities in

TABLE I

RTT-RELATED CALIBRATION PARAMETERS

\begin{tabular}{l|c|c|c|} 
& Intercept $[\mathrm{ns}]$ & Slope $[\mathrm{ns} / \mathrm{cm}]$ & Nominal slope $[\mathrm{ns} / \mathrm{cm}]$ \\
\hline Slave 1 & 858.21 & 0.1445 & 0.0667 \\
\hline Slave 2 & 842.62 & 0.1194 & 0.0667 \\
\hline Slave 3 & 843.60 & 0.0947 & 0.0667 \\
\hline
\end{tabular}

TDCs, dependency on humidity and temperature, sensitivity to artifacts and human bodies and false triggering due to electromagnetic disturbances. Calibration partially overcomes these effects, while signal processing can feature additional mitigation capabilities. As an example, in the proximity of the maximum receiver distance range, the receiving probability drops significantly. However, an eventual undetected pulse results in over-range of the TDC, that can easily be identified. Similarly, by observing the distribution of measured RTTs, outliers can be removed. Outliers are observations that are numerically distant from the rest of data, that would surely be removed from the record by a casual observer. Examples are measurements of master-slave distances being excessively large, because of TDC over-range due to return pulse miss, or excessively small because of the detection of untimely disturbing pulses. Methods to remove these data can be based on apriori information about assumed distribution of RTTs, so that hypothesis testing can then be applied to decide whether the measured RTT is or is not adding information useful for position estimation purposes. Similarly, if a rough position estimate is available along with indications on the speed, as it is when the master position is tracked, by setting an RTT acceptance interval, outlying measurements can be rejected. Similar other constraints can be added to select informative from non-informative pulses.

In processing experimental data, a pragmatic approach has been taken here, to avoid making assumptions on the distribution of complying RTTs. NLS-based estimation has been applied to distance triplets only after removal of outliers. Decision on rejection or approval of each sample in a record of $N$ measured distances between the master and the $i$-th slave, has been taken on the basis of the value of the Mahalanobis distance defined as follows:

$$
\boldsymbol{M D}_{i}=\frac{\left|\boldsymbol{d}_{i}-\mu_{i}\right|}{\sigma_{i}} \quad i=1,2,3
$$

where $\boldsymbol{M} \boldsymbol{D}_{i}$ is an $N$-size column vector, $\boldsymbol{d}_{i}$ is the vector containing the $N$ calibrated distances and $\mu_{i}$ and $\sigma_{i}$ are the corresponding sample average and standard deviation, respectively. Data in $\boldsymbol{M} \boldsymbol{D}_{i}$ are ordered and the higher $p \%$ classified as outliers. If outliers are just removed from further processing the record size decreases. Alternatively, each outlier is substituted by the mean of remaining values. In our system we did not notice a significant difference in the results obtained applying either one of two approaches: while the former leads to an increase in the position estimator variance, the latter provides estimates biased towards the mean. The value of $p$ has been chosen equal to 0.2 as a results of a trade-off choice: if it is too large the RTT Probability Density Function (PDF) is trimmed too much, while if it is too small, too few abnormal data are identified. In Fig. 9 the histograms of $5 \cdot 10^{3}$ RTTs are plotted in a given master-slave configuration, before and after outlier removal has been applied. Notice that both plots have been obtained for similar numbers of bars, that span over different ranges. A fitted Gaussian PDF is also plotted for reference. Estimated standard deviations are 17.35 and $14.20 \mathrm{~cm}$, before and after outlier removal, respectively. This latter figure is of the same order of magnitude of that 
estimated in sec. III using information on pulse rise-time. While the resulting estimated PDF is still asymmetric around the mean, the outlier removal algorithm reduces its skewness and mitigates the effect of markedly uninformative pulses. Moreover, experiments have highlighted heteroscedasticity in the collected RTTs records, the variance being distancedependent. This results in worse estimation accuracy at larger distances from slaves, but could be exploited to improve accuracy of used position estimation algorithms by including this information in the procedure's constraints. Thus, the overall estimation procedure is based on four steps: compensation of offset and slope by means of each node calibration curve, removal of outliers, averaging of $N$ measurement results and application of the NLS algorithm. Similary to the approach taken in [39], Tab. II reports calculations regarding the algorithm computational complexity for a generic setup in which the three spatial coordinates are estimated, by using $S$ slaves and $N$ measurement results for each slave.

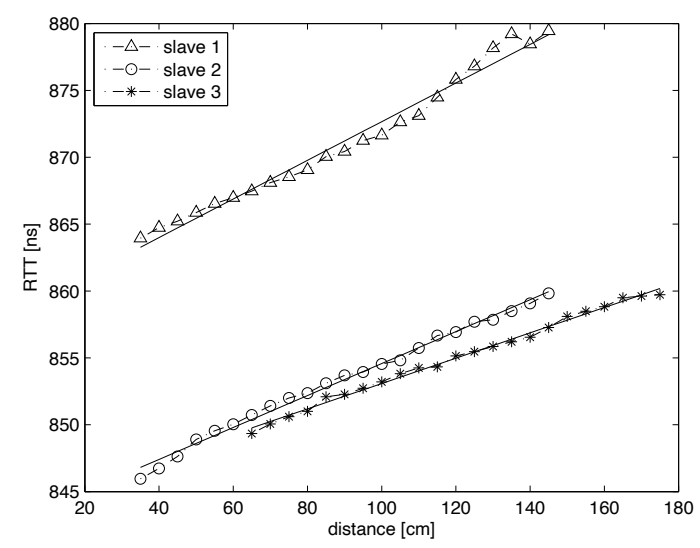

Fig. 8. RTTs of the 3 slaves used in the system under calibration conditions including the corresponding linear fitting.

\section{Position Measurement Results}

The measurement setup includes 3 slaves put at known positions at the vertices of a triangle in a laboratory environment, as shown in Fig. 11. The master has been navigated on a rectangular path and 8 fixed positions have been estimated on the basis of records having size $N=5 \cdot 10^{3}$. For each entry in the record, 3 RTTs have been measured by the TDC, managed by the DCU. Compensation of slope and intercept using calibration data has been applied and outliers removed and substituted by the mean in each record. The effects of the outlier removal procedure are shown in Fig. 10 where the position histograms have been plotted before and after removal obtained using data in one of the records. Finally, the three mean values obtained using $N$-size records modified according to the described outlier removal technique, have been used as the known data for the NLS algorithm to provide position estimations, shown in Fig. 11 using circles. Experimental data show that good matching between known and estimated positions has been achieved, while type-A standard uncertainty [40] in short-distance single measurement
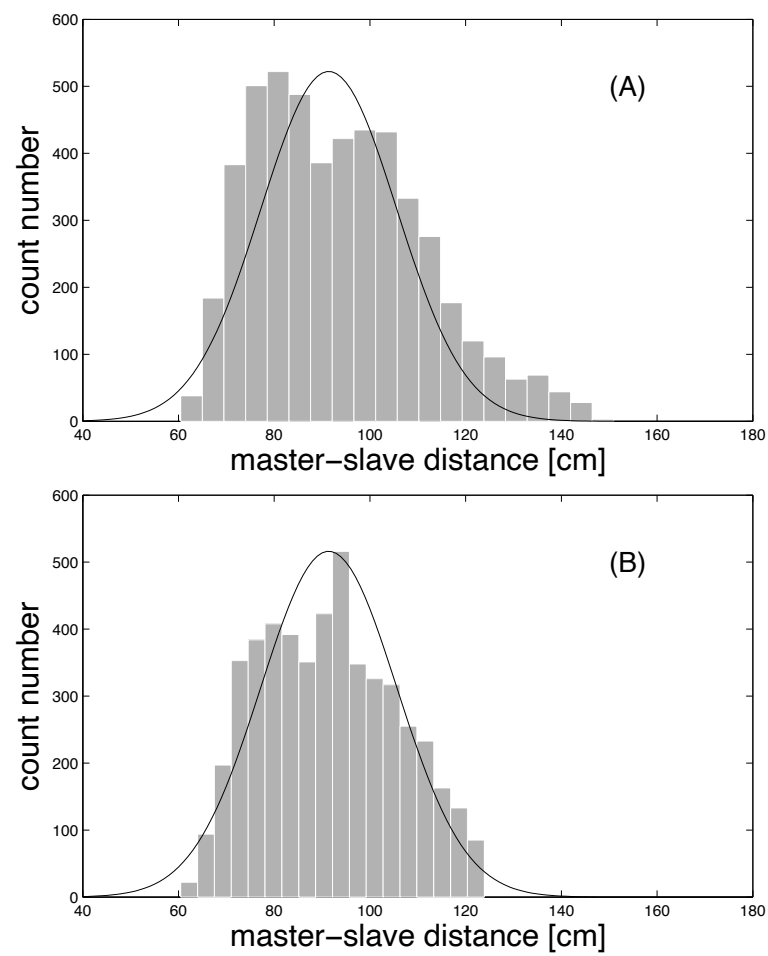

Fig. 9. Histograms of $5 \cdot 10^{3}$ RTTs in a given master-slave configuration, before (A) and after (B) removing outliers. Gaussian fits are shown for reference purposes.

results did not exceed $10 \mathrm{~cm}$, after outlier removal. This result is consistent with the value of the pulse rise-time and comparable with those obtained by other researchers using more complex architectures [15].

\section{AnAlysis of UnCERTAinty Sources}

In this section, we analyze the effects of system non idealities to the uncertainty in the estimation of the master position. Since start and stop triggers originate when the pulse crosses a fixed-value threshold, the shape of the transmitted and received pulses plays a major role in influencing system accuracy and is the dominant source of uncertainty in this realization. The pulse shape is in turn largely dependent on the characteristics of the pulse generator, on the amount of wideband noise, on multipath induced effects, on path-losses and on deviations and tolerances in the fixed frequencies used to drive the mixer. The high-slew rate properties of the UWB pulse, make it rather robust against time-local disturbances, but its time-dependent derivative is the main reason for the measured RTTs not to exhibit a symmetric distribution. In fact, wideband noise superimposed on the pulse signal, makes the probabilities of being above or below the set threshold asymmetric. As a consequence, the estimator of the RTT probability density function applied to experimental data obtained at several master-slaves distances confirm the leptokurtic behavior of the RTTs distributions.

Multipath effects play also a role, both in determining the pulse shape and in influencing RTT measurements. It is often argued that UWB-based transmission is resilient to multipath 
TABLE II

COMPUTATIONAL COMPLEXITY OF THE POSITION ESTIMATION ALGORITHM.

\begin{tabular}{|c|c|c|c|c|c|}
\hline & Multiplications/divisions & Additions/subtractions & Sorting operations & Transpose operations & Inverse operations \\
\hline Calibration & $N \times S$ & $N \times S$ & - & - & - \\
\hline Outlier rejection & $N \times S$ & $N \times S$ & $S$ & - & - \\
\hline Data averaging & $S$ & $(N-1) \times S$ & - & - & - \\
\hline $\mathrm{LS}$ & $21 S$ & $6(3 S-2)$ & - & ]$_{S \times 3}$ & ]$_{3 \times 3}$ \\
\hline NLS & \multicolumn{5}{|c|}{$\begin{array}{l}\text { The NLS algorithm is first based on a Jacobian operation to linearize the nonlinear position equations. Then the } \\
\text { LS equations can be used to estimate the position. Therefore, the computational complexity of NLS is as follows: } \\
\text { \# NLS cycles } \times \text { (computational complexity of LS + Jacobian operation). }\end{array}$} \\
\hline
\end{tabular}

$N=$ number of measurement results for each slave, $S=$ number of slaves.
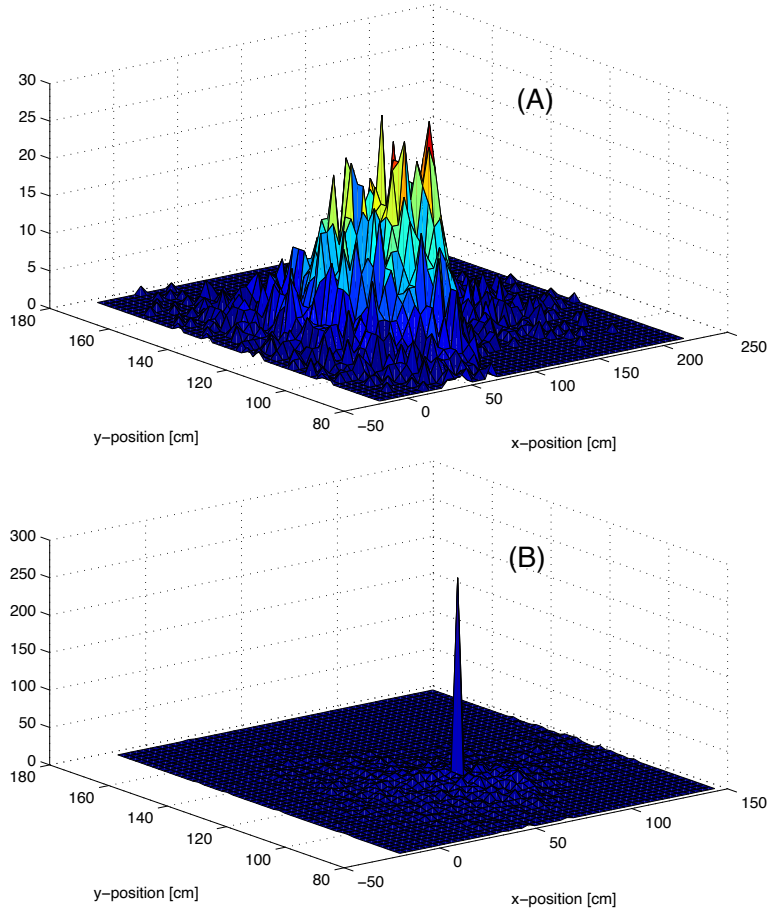

Fig. 10. Two-dimensional histograms of $5 \cdot 10^{3}$ estimated position coordinates obtained before (A) and after (B) removing outliers.

effects. Of course, reflected RF pulses arrive at a later time at the receiver with respect to the line-of-sight pulse, so that their contribution might seem uninfluencial in determining the RTT. However, closely time-spaced replica of the pulse at the receiver, that may happen in a laboratory setup, may modify the shape and slopes in the received signal and thus influence measurement results. In order to verify this hypothesis, experimental data have also been collected in a semianechoic chamber, where multipath phenomena can largely be neglected. The demodulated pulses show markedly different behaviors. When reflections are not permitted, the pulse is a small-energy compact support waveform. On the contrary, in the laboratory environment, reflections in the walls and other artifacts result in a longer transient behavior of the pulse, that is also characterized by a larger energy content. In both cases, repeatability of measurements is confirmed by similar values in RTT standard deviations, but different calibration curves apply because of different experimental conditions. At

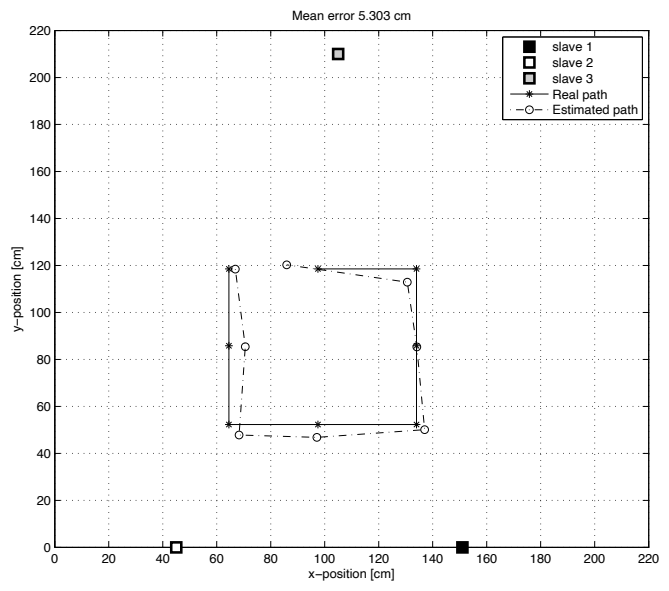

Fig. 11. Final position estimation results: squares indicate the position of the slaves, black dots the nominal position of the master, circles the estimated positions of the master.

the same time, the laboratory setup guarantees an extended range of transmission over that obtained in the semi-anechoic chamber because of the wave-guide effect of walls and of the resulting constructive interference induced by multipath. Sample distributions of measured RTTs have been processed in both environments, exhibiting similar overall behaviors. However, since multipath is a highly position dependent phenomenon [41], its effects must be taken into consideration if an environmentally-independent positioning system is a design requirement. Deviations in the initial phase and frequencies used in the modulators and demodulators, to translate the pulse in the transmitters and receivers, reduces the received pulse amplitudes in the nodes and the related detection probability, with respect to what happens under ideal conditions. Fine tuning or more involved hardware architectures could mitigate the effects of this phenomenon at the expense of an increased complexity. It is also to be observed that a missed pulse can be detected by putting simple constraints on the RTT measured by the TDCs, such as when detecting over-range events.

Wideband noise and path-loss effects contribute to both reduce the SNR at the receiver side and to attenuate the received signal. As an overall result, an increased standard deviation is expected at larger distances. This phenomenon is evidenced in Fig. 12, where estimated standard deviations in RTTs is shown as a function of the master-slave distance 


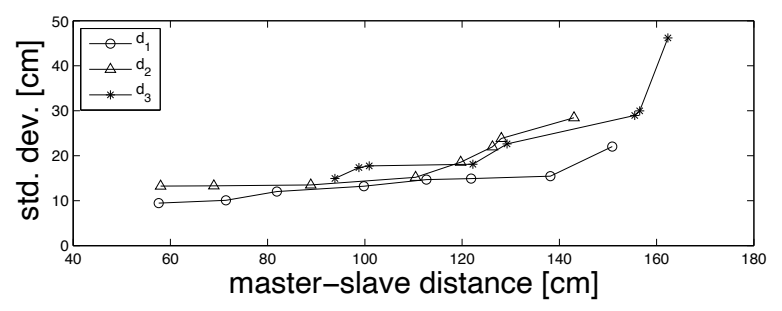

Fig. 12. Behavior of standard deviation as a function of the distance between master and slave devices, for each of the three nodes in the position measurement system.

between the three nodes in the system. The almost linearly increasing behavior is the evidence of the heteroschedastic characteristic of RTTs, mentioned in section III and represents an experimental estimate of the overall uncertainty to be attributed to single RTT measurements. Data in Fig. 12 are not processed for outliers removal. Conversely, lower uncertainties result, as proved by data shown in Fig. 10.

\section{CONCLUSION}

In this paper we described the design and measurement results of a position estimation system working at $5.6 \mathrm{GHz}$, entirely realized using COTS components. Result have been obtained both in a laboratory and in a semi-anechoic chamber environments, but their applicability includes outdoor areas as well. Good accuracy has been achieved, by maintaining lowcomplexity and low-costs.

Difficulties to be overcome in this research require transdisciplinary approaches at system, device and signal processing levels, whose implications have been highlighted in this paper. The whole process has been described also to allow easy system upgrade when advances in design and technology will provide devices with improved performances. Owing to its simplicity, to the usage of UWB pulses and to the reduced number of devices in the signal path, this system can also be employed to achieve clock synchronization in the nodes of a sensor network, given that the pulse time-of-flight can often be neglected in typical applications within this field [42].

\section{ACKNOWLEDGMENT}

This research has been supported by MIUR grant PRIN 2008TK5B55-2008.

\section{REFERENCES}

[1] C. Zhang, M. J. Kuhn, B. C. Merkl, A. E. Fathy, and M. R. Mahfouz, "Real-time noncoherent uwb positioning radar with millimeter range accuracy: Theory and experiment," IEEE Trans. on Microwave Theory and Techniques, vol. 58, no. 1, pp. 9-20, Jan. 2010.

[2] K. Pahalavan, L. Xinrong, and J. P. Makela, "Indoor geolocation science and technology," IEEE Communications Magazine, vol. 40, no. 2, pp. 112-118, Feb. 2002.

[3] H. Liu, H. Darabi, P. Banerjee, and J. Liu, "Survey of wireless indoor positioning techniques and systems," IEEE Transactions on Systems, Man, and Cybernetics, Part C: Applications and Reviews, vol. 37, pp. 1067-1080, Nov. 2007.

[4] R. Fontana, "Recent system applications of short-pulse ultrawideband (uwb) technology," IEEE Transactions on Microwave Theory and Techniques, vol. 52, pp. 2087-2104, Sept. 2004.

[5] T. Kaiser, A. Molisch, M. D. Benedetto, I. Oppermann, and D. Porcino, "Uwb communication systems: A comprehensive overview," Hindawi Publishing Corporation, Sept. 2006.
[6] H. Mextorf and F. Daschner and M. Kent and R.H. Knöchel, "UWB Free-Space Characterization and Shape Recognition of Dielectric Objects Using Statistical Methods," IEEE Trans. Instr. Meas., vol. 60, no. 4 , pp. 1389-1396, April 2011.

[7] A. Molish, "Ultrawideband propagation channels-theory, measurement, and modeling," IEEE Transactions on Vehicular Technology, vol. 54, pp. 1582-1545, Sept. 2005.

[8] M. R. Mahfouz and M. J. Kuhn, "Uwb channel measurements and modeling for positioning and communications systems in the operating room," Proc. of the IEEE Topical Conf. on Biomedical Wireless Technologies, Networks \& Sensing Systems (BioWireleSS), pp. 47-50, Feb. 2011.

[9] E. Plouhinec and B. Uguen, "Ray-tracing correction for through-thewall propagation: Application to uwb indoor positioning," Proc. of the 2009 IEEE Intern. Conf. on Utra-Wideband (ICUWB2009), pp. 240 244, Sept. 2009.

[10] J. Xu, M. Ma, and C. L. Law, "Theoretical lower bount for uwb tdoa positioning," Proc. of the 2007 IEEE Global Telecommunicaition Conference (GLOBECOM07), pp. 4101-4105, Dec. 2007.

[11] J. K. Zaleski, T. Yamazato, and M. Katayama, "Experimental validation of toa uwb positioning with two receivers using known indoor features," Proc. of the 2010 IEEE/ION Position Location and Navigation Symposium (PLANS), pp. 505-509, July 2010.

[12] Zhou Yuan and Choi Look Law and Yong Liang Guan and F. Chin, "Indoor Elliptical Localization Based on Asynchronous UWB Range Measurement," IEEE Trans. Instr. Meas., vol. 60, no. 1, pp. 248 - 257, Jan. 2011.

[13] M. Demirkan and R. R. Spencer, "A pulse-based ultra-wideband transmitter in 90-nm cmos for wpans," IEEE Journal of Solid-State Circuits, vol. 43, no. 12, pp. 2820-2828, Dec. 2008.

[14] M. R. Mahfouz, A. E. Fathy, M. J. Kuhn, and Y. Wang, "Recent trends and advances in uwb positioning," IEEE MTT-S International Microwave Workshop on Wireless Sensing, Local Positioning, and RFID (IMWS 2009), pp. 1-4, Sept. 2009.

[15] C. Meier, A. Terzis, and S. Lindenmeier, "A robust 3d high precision radio location system," IEEE MTT-S Int. Microw. Symp. Dig., pp. 397400, 2007.

[16] A. Fujii, H. Sekiguchi, M. Asai, S. Kurashima, H. Ochiai, and R. Kohno, "Impulse radio uwb positioning system," IEEE Radio Wireless Symp., 2007, pp. 55-58, 2007.

[17] Y. Depeng, L. Husheng, G. D. Peterson, and A. Fathy, "Compressive sensing tdoa for uwb positioning systems," IEEE Radio Wireless Symp., 2007, pp. 194-197, 2011.

[18] Y. Zhou and C.L. Law and F. Chin, "Construction of Local Anchor Map for Indoor Position Measurement System," IEEE Trans. Instr. Meas., vol. 59, no. 7, pp. 1986-1988, July 2010.

[19] X. Sun, Y. Ma, J. Xu, J. Zhang, and J. Wang, "A high accuracy monostation uwb positioning system," Proc. of the 2008 IEEE Intern. Conf. on Utra-Wideband (ICUWB2008), vol. 1, pp. 201-204, Dec. 2008.

[20] Ubisense, 2011. [Online]. Available: http://www.ubisens.net

[21] A. D. Angelis, M. Dionigi, A. Moschitta, R. Giglietti, and P. Carbone, "Characterization and modeling of an experimental uwb pulse-based distance measurement system," IEEE Trans. on Instrumentation and Measurements, vol. 58, no. 5, pp. 1479-1486, May 2009.

[22] I. Gresham and A. Jenkins and R. Egri and C. Eswarappa and N. Kinayman and N. Jain and R. Anderson and F. Kolak and R. Wohlert and S.P. Bawell and J. Bennett and J.P. Lanteri, "Ultra-wideband radar sensor for short-range vehicular applications," IEEE Trans. Microwave Theory, vol. 52, no. 9, pp. 2105-2122, 2004.

[23] G. Turin, "An introduction to matched filters," IRE Trans. Inf. Theory, vol. 6, no. 3, pp. 311-329, June 1960.

[24] Dual 2-Input Positive AND Gate SN74LVC2G08, Texas Instruments, 2007. [Online]. Available: http://www.ti.com

[25] Dual Inverter Gate SN74LVC2G04, Texas Instruments, 2007. [Online]. Available: http://www.ti.com

[26] HMC358ms8G: Voltage Controlled Oscillator, Hittite Microwave Corporation, 2010. [Online]. Available: http://www.hittite.com

[27] HMC220ms8: Double Balanced Mixer, Hittite Microwave Corporation, 2010. [Online]. Available: http://www.hittite.com

[28] HMC407ms8G: InGaP HBT Power Amplifier SMT, Hittite Microwave Corporation, 2006. [Online]. Available: http://www.hittite.com

[29] HMC320ms8:GaAs MMIC Low Noise Amplifer SMT, Hittite Microwave Corporation, 2009. [Online]. Available: http://www.hittite.com

[30] SNA386:GaAs MMIC Amplifier, Sirenza Microdevices, 2001. [Online]. Available: http://www.sirenza.com

[31] LTC5536:Precision RF Detector with Fast Comparator Output, Linear Technology, 2006. [Online]. Available: http://www.linear.com 
[32] TDC-GP2: Time to Digital Converter, Acam, 2006. [Online]. Available: http://www.acam.com

[33] Christoph Mittermayer and Andreas Steininger, "On the Determination of Dynamic Errors for Rise Time Measurement with an Oscilloscope," IEEE Trans. Instr. Meas., vol. 48, no. 6, pp. 1103 - 1107, Dec. 1999.

[34] J. Liang, C. Chian, X. Chen, and C. Parini, "Study of a printed circular disc monopole antenna for uwb systems," IEEE Trans. Antennas Propagat., vol. 53, pp. 3500-3504, Nov. 2005.

[35] M. Dionigi and M. Mongiardo, "Design of via hole fed printed circular disc monopole antenna for uwb systems," IEEE-APS Topical Conference on Antennas and Propagation in Wireless Communications (IEEE $A P W C$ ), Oct. 2011

[36] J. H. Bryant, Principles of Microwave Measurements. Institution of Electrical Engineers, 1993.

[37] J. O. Nilsson, A. D. Angelis, I. Skog, P. Carbone, and P. Händel, "Signal processing issues in indoor positioning by ultra wide band radio aided inertial navigation," 17th European Signal Processing Conference (EUSIPCO 2009), Aug. 2009.

[38] S. M. Kay, Fundamentals of Statistical Signal Processing, Volume I: Estimation Theory. Upper Saddle River, New Jersey, USA: Prentice Hall, 2001.

[39] Y. S. Chiou and C. L. Wang and S. C. Yeh, "An adaptive location estimator using tracking algorithms for indoor WLANs," Wireless Networks, no. 16, pp. 1997-2012, 2010.

[40] Joint Committee for Guides in Metrology. (2008, September) Evaluation of Measurement Data - Guide to the Expression of Uncertainty in Measurement. [Online]. Available: http://www.bipm.org

[41] A. A. M. Saleh and R. A. Valenzuela, "A Statistical Model for Indoor Multipath Propagation," IEEE Journal on Selected Areas in Communications, vol. SAC-5, no. 2, pp. 128-137, Feb. 1987.

[42] A. Flammini, C. M. D. Dominicis, S. Rinaldi, E. Sisinni, A. Cazzorla, A. Moschitta, and P. Carbone, "High-precision uwb-based timestamping," 2011 International IEEE Symposium on Precision Clock Synchronization for Measurement, Control and Communication, Sept. 2011. 